\title{
F-actin cytoskeleton reorganization is associated with hepatic stellate cell activation
}

\author{
XIAODONG CUI ${ }^{*}$, XIAOYUN ZHANG*, QINGLING YIN*, AIXIA MENG, SHAOJUAN SU, \\ XU JING, HONG LI, XIUMEI GUAN, XIN LI, SHUNMEI LIU and MIN CHENG \\ Medical Research Center, Weifang Medical University, Weifang, Shandong 261053, P.R. China
}

Received September 10, 2013; Accepted February 20, 2014

DOI: $10.3892 / \mathrm{mmr} .2014 .2036$

\begin{abstract}
The activation of hepatic stellate cells (HSCs) is involved in the development of hepatic fibrosis. Previous studies have indicated that the acquisition of certain properties by activated HSCs is highly dependent on the reorganization of the actin cytoskeleton. However, direct evidence showing that the reorganization of the actin cytoskeleton is responsible for HSC activation is lacking. The aim of the present study was to investigate the role of cytoskeletal reorganization during HSC activation and to clarify the underlying mechanism. HSC-T6 cells were treated either with the F-actin stabilizer jasplakinolide (Jas) or the depolymerizer cytochalasin D (Cyto D). The actin cytoskeleton was evaluated via assessment of stress fiber formation. Furthermore, the activation properties of HSCs, including proliferation, adhesion, migration and the expression of $\alpha$-smooth muscle actin ( $\alpha$-SMA) and collagen 1 , were investigated in vitro. The results showed that Jas and Cyto D affected the actin distribution in HSC-T6 cells. Treatment with Jas resulted in thick actin bundles and a patchy appearance in the cytoplasm in HSC-T6 cells. In parallel, polymerization of actin microfilaments induced by Jas upregulated the expression of $\alpha$-SMA and collagen 1 , and also enhanced the migration and adhesion properties of HSC-T6 cells. Furthermore, the activation of HSC-T6 cells induced by the reorganization of the actin cytoskeleton was associated with the p38 mitogen-activated protein kinase (p38 MAPK) pathway. In conclusion, the present study suggests that the reorganization of the F-actin cytoskeleton is associated with HSC activation and that the p38 MAPK pathway is involved in this process. The inhibition of F-actin reorganiza-
\end{abstract}

Correspondence to: Dr Min Cheng, Medical Research Center, Weifang Medical University, No. 7166 Baotong Street, Weifang, Shandong 261053, P.R. China

E-mail: mincheng@wfmc.edu.cn

*Contributed equally

Key words: hepatic stellate cells, F-actin, cytoskeleton, migration, adhesion, gene expression tion may thus be a potential key factor or molecular target for the control of liver fibrosis or cirrhosis.

\section{Introduction}

It has been reported that hepatic stellate cells (HSCs) are involved in the development of hepatic fibrosis and in cancer cell invasiveness (1-3). Under physiological conditions, HSCs are quiescent and have important roles in the regulation of retinoid homeostasis and extracellular matrix (ECM) remodeling. However, when the liver is damaged by certain factors, including viral infection, chronic alcohol abuse and inflammation, quiescent HSCs undergo a process of activation that is characterized by trans-differentiation into $\alpha$-smooth muscle actin ( $\alpha$-SMA)-positive myofibroblast-like cells, and produce a large quantity of ECM components, including collagen types 1 and 3, as well as other matrix proteins. The fibrogenic features of HSCs, together with an induced ability to synthesize and deposit ECM components, represent a key cellular event in the genesis of liver cirrhosis.

The cytoskeleton is accountable for a variety of physiological events in the cell, including the formation of stress fibers, adhesion, migration, apoptosis and receptor clustering in different cell models $(4,5)$. A study by Yee (6) indicated that the activation of HSCs is accompanied by changes in the cellular cytoskeleton. As a highly conserved protein, actin constitutes an essential component of the cytoskeleton in most cells and exists in two principal forms: Globular monomeric $(\mathrm{G})$ and filamentous polymeric $(\mathrm{F})$. G-actin molecules are soluble in diluted salt solution and polymerize into F-actin when their concentration is increased. In culture, HSC activation can be distinguished by the development of prominent cytoplasmic fibers, the loss of perinuclear droplets and cell spreading (i.e., increasing in size). This cytoskeletal reorganization provides the driving force for cell movement and surface remodeling (7). Based on these observations, it was hypothesized in the present study that the actin cytoskeleton is directly involved in the morphological and functional changes in HSCs that are associated with activation. HSC-T6 is a rat hepatic stellate cell line (8) derived from primary HSCs as an in vitro assay system. For the establishment of this cell line, the primary HSCs were transformed with the simian virus 40 large T-antigen and a stable phenotype exhibiting an activated phenotype with a fibroblast-like shape and high 
proliferation activity was established (9). It is considered that the immortalized cells are likely to prove useful in exploring the key process involved in hepatic fibrogenesis. To evaluate this hypothesis, the HSC-T6 cells were treated with either the F-actin stabilizer jasplakinolide (Jas) or the depolymerizer cytochalasin D (Cyto D). The actin cytoskeleton was then evaluated by assessment of stress fiber formation in HSCs. In the present study, the effects of the cytoskeletal reorganization induced by Jas or Cyto D on the activation of HSCs were investigated using a variety of experimental tools.

\section{Materials and methods}

Cell culture. HSC-T6 cells, a spontaneously immortalized rat HSC line, were purchased from the Cell Bank of Xiangya School of Medicine (Changsha, China), and maintained in high-glucose Dulbecco's Modified Eagle medium (DMEM; Gibco $^{\circledR}$; Invitrogen Life Technologies, Carlsbad, CA, USA) supplemented with $15 \%(\mathrm{v} / \mathrm{v})$ fetal bovine serum (FBS; HyClone, Waltham, MA, USA). The study was approved by the Ethics Committee of Weifang Medical University (Weifang, China; permit no. 2013024).

Cytoskeleton staining. Following being serum-starved for $12 \mathrm{~h}$, HSC-T6 cells were treated with Jas (100 nmol/l) (10) or Cyto D (1 $\mu \mathrm{mol} / \mathrm{l})$ (11) for $30 \mathrm{~min}$. Corresponding control groups received equal volumes of dimethylsulfoxide (DMSO). Following being fixed with $4 \%$ paraformaldehyde at $4{ }^{\circ} \mathrm{C}$ for $30 \mathrm{~min}$, cells were stained with $1.0 \mu \mathrm{g} / \mathrm{ml}$ phalloidin-fluorescein isothiocyanate (FITC) (Enzo Life Sciences, Alexis Biochemicals, San Diego, CA, USA) for $40 \mathrm{~min}$ at room temperature. The images were acquired using a fluorescence microscope (Leica, Mannheim, Germany).

Cell proliferation assay. The 5'-ethynyl-2'-deoxyuridine (EdU) incorporation assay was performed to quantify cell proliferation according to the manufacturer's instructions (Guangzhou Ribobio Co., Ltd, Guangzhou, China). More than five random fields per well were captured (magnification, x100) and Image-Pro Plus 6.0 (Media Cybernetics, Inc., Rockville, MD, USA) was used to calculate the percentage of EdU-positive cells identified by Apollo ${ }^{\circledR} 567$ fluorescence in the total cells identified by Hoechst 33342 nuclear staining.

Cell proliferation was also examined using the cell counting kit-8 (CCK-8, Dojindo Molecular Technologies, Kumamoto, Japan). HSC-T6 cells (1x10 4 cells/well) were seeded in 96-well plates and incubated overnight in DMEM containing $10 \%$ FBS. The cells were then transferred to serum-free conditions for $12 \mathrm{~h}$. Following treatment with Jas or Cyto D, $100 \mu \mathrm{l}$ medium containing cell counting kit- 8 was added to the cells in the 96 -well plates, which were subsequently incubated for $2 \mathrm{~h}$ at $37^{\circ} \mathrm{C}$. The absorbance at $450 \mathrm{~nm}$ was determined using a multi-plate reader (Lambda Bio-20; Beckman Coulter, Inc., Brea, CA, USA).

Cell adhesion assay. Cells were trypsinized and resuspended in serum-free media containing $0.25 \%$ bovine serum albumin. Equal numbers of cells were seeded onto the plates and incubated for $1 \mathrm{~h}$ at $37^{\circ} \mathrm{C}$. Following the removal of non-adherent cells by washing, adherent cells were counted independently in six random, high-power microscope fields (HPFs) (magnification, x100)/well by three observers blinded to the treatments.

Cell migration assay. A modified Boyden chamber (Costar, Cambridge, MA, USA) assay was used to evaluate the migratory function of cells. Briefly, a total of $1 \times 10^{5}$ HSC-T6 cells were placed in the upper chamber, while the medium was placed in the lower chamber. The assays were conducted over a 16 -h incubation period at $37^{\circ} \mathrm{C}$ in an incubator equilibrated with $5 \% \mathrm{CO}_{2}$. The membrane was then gently washed with PBS and fixed with $4 \%$ paraformaldehyde. Non-migrating cells were gently removed with cotton balls from the upper side of the membrane, and the membrane was then stained with DAPI. The migration of late HSCs was evaluated by counting the migrated cells in six random HPFs (magnification, x100)/well.

Cell apoptosis assay. HSC-T6 cells $\left(1 \times 10^{6}\right)$ were stained with annexin V-FITC and propidium iodide (PI) (BD Biosciences, Franklin Lakes, NJ, USA). Following staining, the cells were washed twice with binding buffer. Apoptotic cells were detected by fluorescence-activated cell sorting (FACS). Fluorescence parameters were gated using unstained and single-stained cells, and 20,000 cells were collected for each sample. Apoptotic percentage analysis was performed using CellQuest ${ }^{\mathrm{TM}}$ software (BD Biosciences).

$R N A$ isolation and quantitative polymerase chain reaction (qPCR). Total cellular RNA was isolated using TRIzol ${ }^{\circledR}$ reagent (Invitrogen Life Technologies) and reverse-transcribed into cDNA using the SYBR ${ }^{\circledR}$ PrimeScript ${ }^{\circledR}$ RT-PCR kit (Takara Bio, Inc., Shiga, Japan) at $37^{\circ} \mathrm{C}$ for $15 \mathrm{~min}$. Gene expression was evaluated using SYBR ${ }^{\circledR}$ Premix Ex Taq ${ }^{\mathrm{TM}}$ (Takara). The rat $\alpha$-SMA sequences were: Forward, AGCCAGTCGCCATCA GGAAC, and reverse, CCGGAGCCATTGTCACACAC. The collagen type 1 sequences were: Forward, GACATG TTCAGCTTTGTGGACCTC, and reverse, AGGGAC CCTTAGGCCATTGTG. GAPDH was used as an internal control, and the sequences were: Forward, GGC ACAGTCAAGGCTGAGAATG, and reverse, ATGGTG GTGAAGACGCCAGTA. The thermal cycling conditions were as follows: $30 \mathrm{sec}$ at $95^{\circ} \mathrm{C}$ for pre-denaturation, followed by 42 cycles of $15 \mathrm{sec}$ at $95^{\circ} \mathrm{C}$ for denaturation, $1 \mathrm{~min}$ at $59^{\circ} \mathrm{C}$ for annealing and $10 \mathrm{sec}$ at $72^{\circ} \mathrm{C}$ for elongation. At the end of each cycle, the fluorescence emitted by SYBR ${ }^{\circledR}$ Green I was measured. Following completion of the cycling process, samples were immediately subjected to a temperature ramp for melting curve analysis. Relative gene expression was analyzed with the comparative $\mathrm{Ct}$ method $\left(2^{-\Delta \Delta \mathrm{Ct}}\right)$.

Western blot analysis. Proteins were subjected to $12 \%$ SDS-PAGE and then transferred onto a polyvinylidene fluoride membrane. Following blocking in 5\% milk in Tris-buffered saline with Tween 20 (TBST), the membranes were treated with primary antibodies against $\alpha$-SMA (Sigma, St.Louis, MO,USA), collagen type 1 (Santa Cruz Biotechnology, Inc., Santa Cruz, CA, USA) and phosphorylated-p38 mitogen-activated protein kinase (phospho-p38 MAPK; Cell Signaling Technology, Inc., Danvers, MA, USA; 1:100 dilution). GAPDH (Santa Cruz, USA) or p-38 MAPK (Cell Signaling Technology, Inc., Danvers, MA, 

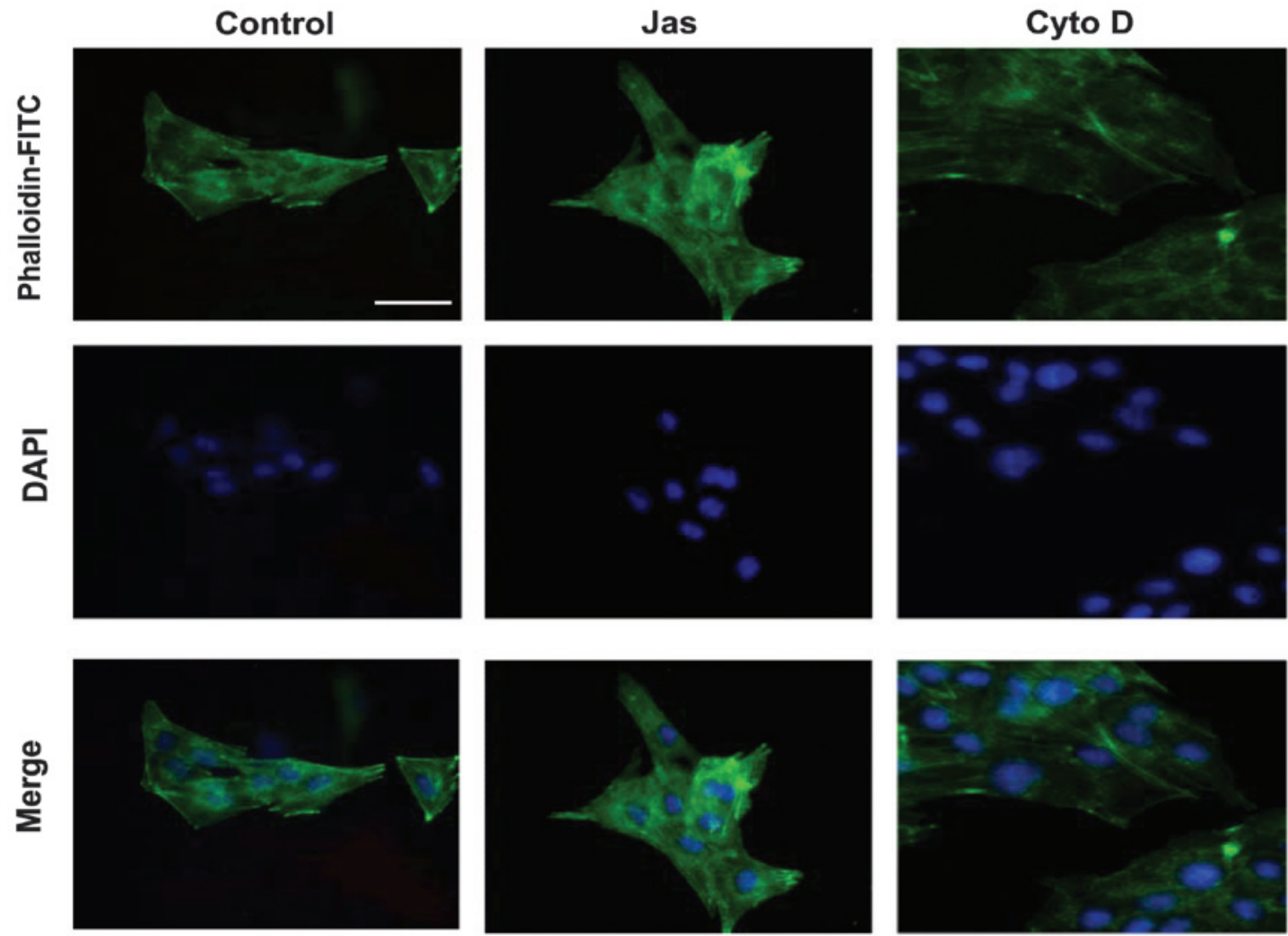

Figure 1. Effects of Jas or Cyto D on the actin cytoskeleton reorganization in HSC-T6 cells. HSC-T6 cells were incubated with dimethylsulfoxide (vehicle control), Jas ( $100 \mathrm{nmol} / \mathrm{l})$ or Cyto D $(1 \mu \mathrm{mol} / \mathrm{l})$, respectively, for $1 \mathrm{~h}$ prior to being fixed in $4 \%$ (v/v) paraformaldehyde for $15 \mathrm{~min}$. The actin filaments were then stained with phalloidin-FITC and the nuclei with DAPI. Images were captured using a fluorescence microscope. Scale bar, $50 \mu \mathrm{m} ;$ magnification, x400. FITC, fluorescein isothiocyanate; Cyto D, cytochalasin D; Ja, jasplakinolide.

USA, 1:100 dilution) were used as control measures. Membranes were then washed with TBST and incubated with secondary antibody conjugated to horseradish peroxidase (Santa Cruz Biotechnology, Inc., 1:2,000 dilution). Immunoreactive bands were visualized by enhanced chemiluminenscence (Amersham Pharmacia Biotech, Amersham, UK), and the resulting autoradiograms were analyzed by densitometry.

Statistical analysis. Unless otherwise indicated, results are expressed as the mean \pm standard error from 3-5 independent experiments. Statistical analyses were performed using one-way analysis of variance, followed by Tukey's test for inter-group comparisons. $\mathrm{P}<0.05$ was considered to indicate a statistically significant difference. All data were analyzed using SPSS 15.0 software (SPSS, Inc., Chicago, IL, USA).

\section{Results}

Effects of Jas or Cyto D on the actin cytoskeleton reorganization in HSC-T6 cells. To evaluate the effects of Jas or Cyto D on the actin reorganization in HSC-T6 cells, the distribution of stress fibers, which can be easily detected by phalloidin, was observed under the fluorescence microscope. The distribution of F-actin in DMSO (vehicle control)-treated HSC-T6 cells exhibited a small network of parallel stress fibers. Treatment with $100 \mathrm{nmol} / \mathrm{l}$ Jas for $1 \mathrm{~h}$ resulted in thick actin bundles and a patchy appearance in the cytoplasm. By contrast, Cyto D ( $1 \mu \mathrm{mol} / \mathrm{l})$-treated cells typically exhibited dissolution of actin stress fibers and decreased fluorescent staining (Fig. 1).
Comparative effect of the reorganization of the actin cytoskeleton induced by Jas or Cyto D on HSC-T6 cell functions. The effects of the actin cytoskeleton reorganization induced by Jas or Cyto D on the HSC-T6 cell functions were investigated in a series of studies (Fig. 2). Initially, cell proliferation was evaluated using the CCK- 8 and EdU incorporation assays. Compared with the DMSO-treated HSC-T6 cells, cell proliferation was decreased in the Cyto D-treated group. However, exposure to Jas did not significantly affect the proliferative activity of HSC-T6 cells (Fig. 2A and B). Furthermore, the adhesion and migration of HSC-T6 cells were determined using the adhesion assay and a modified Boyden chamber, respectively. The results showed that Jas increases, but Cyto D impairs, the adhesion and migration of HSC-T6 cells (Fig. 2C and D). Additionally, apoptotic cells (annexin $\mathrm{V}^{+} / \mathrm{PI}^{-}$) were detected by FACS. The percentages of apoptotic HSC-T6 cells in the Jas- or Cyto D-treated groups were observed to be similar to those in the DMSO-treated group (Fig. 2E).

Effect of the reorganization of the actin cytoskeleton induced by Jas or Cyto D on the activation of HSC-T6 cells. Increased expression levels of $\alpha$-SMA and collagen type 1 are considered to be the major markers of HSC activation $(12,13)$. Compared with the control group, treatment with Jas increased the mRNA levels of $\alpha$-SMA and collagen type 1 . By contrast, the gene expression of $\alpha$-SMA in the Cyto D-treated group was lower than that in the control group (Fig. 3A). Similar results were obtained for the protein levels (Fig. 3B). 
A
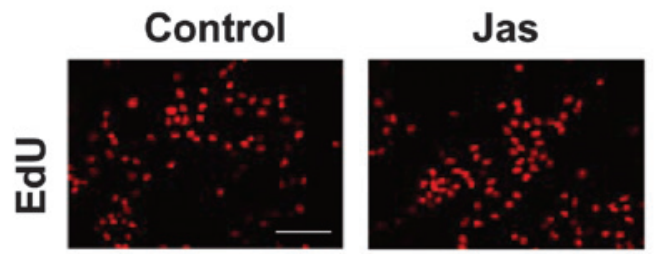

Cyto D
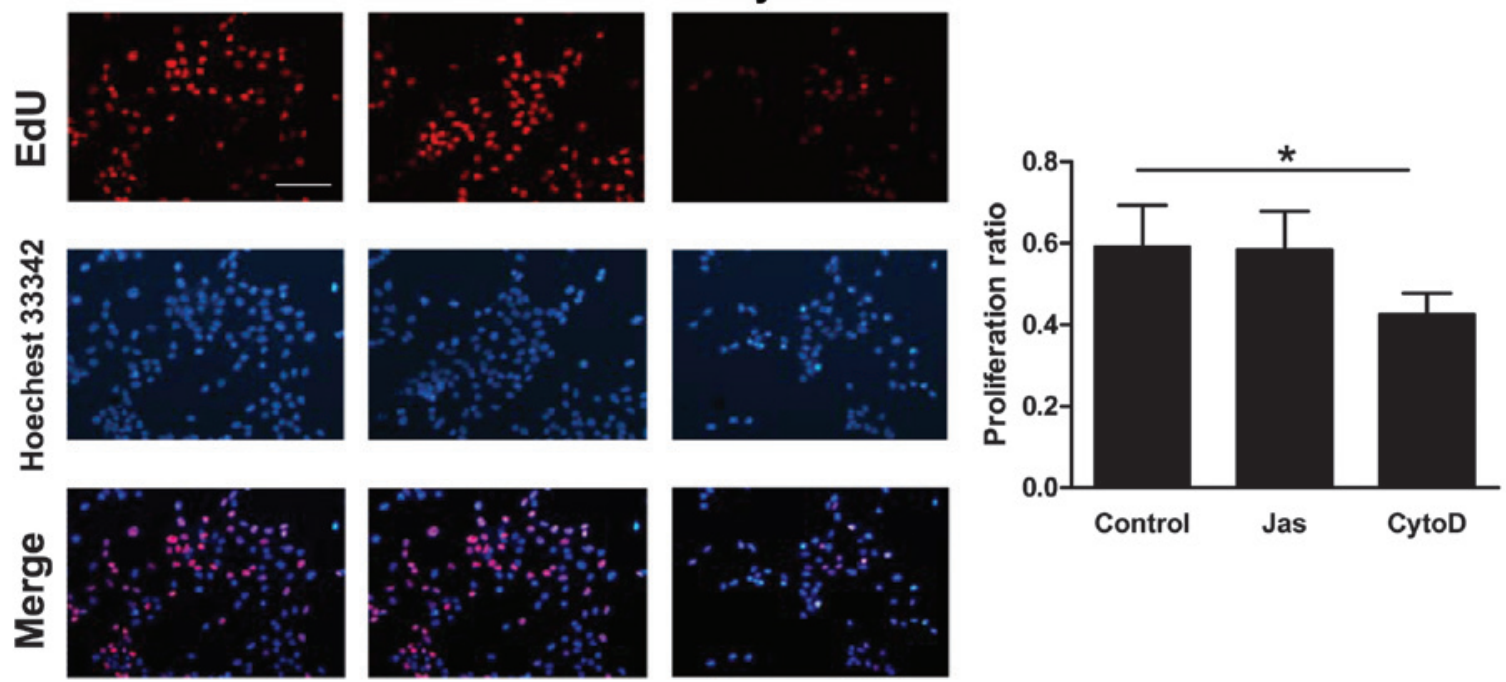

B

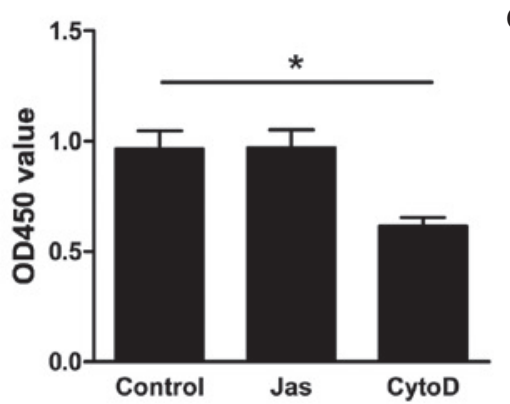

C

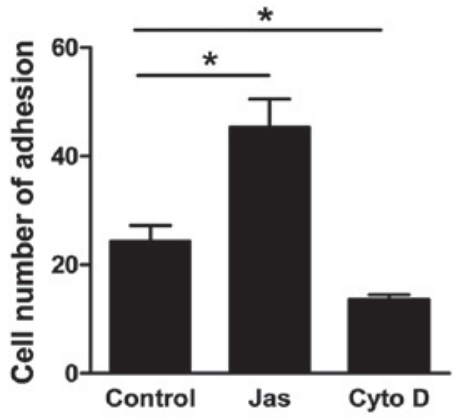

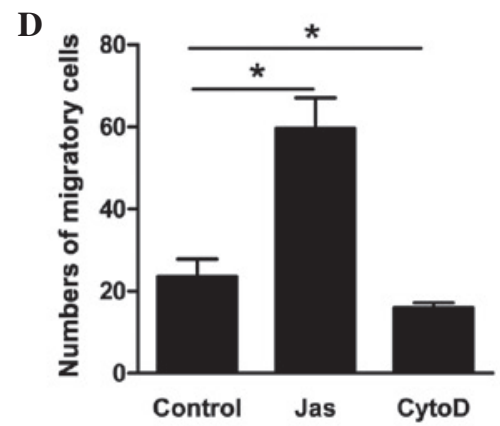

$\mathbf{E}$

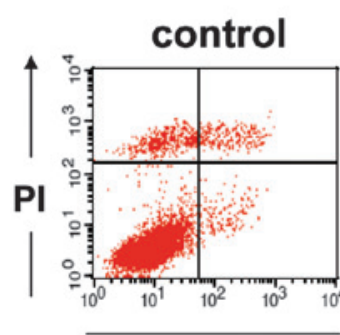

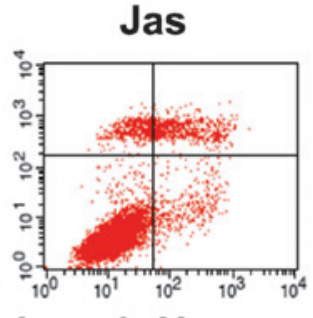

Annexin V
Cyto D

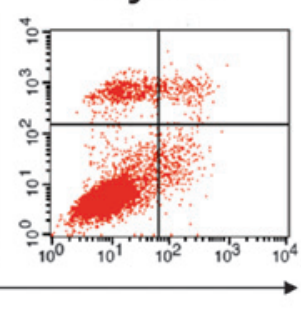

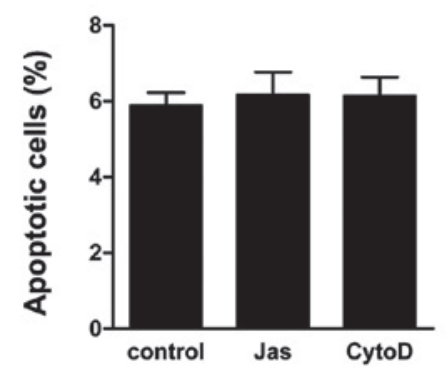

Figure 2. Comparative effect of actin cytoskeleton reorganization induced by Jas or Cyto D on HSC-T6 cell functions. (A) HSC-T6 cells were incubated with DMSO, Jas $(100 \mathrm{nmol} / \mathrm{l})$ or Cyto D $(1 \mu \mathrm{mol} / \mathrm{l})$, respectively, for $1 \mathrm{~h}$. Cells were then washed and cultured in fresh medium for $12 \mathrm{~h}$. Cell proliferation was detected by the EdU incorporation assay. At least seven random fields from each well were captured (scale bar, $100 \mu \mathrm{m}$, magnification, x100), and Image-Pro Plus 6.0 was then used to calculate the percentage of EdU-positive cells in the sample. (B) Cell proliferation was assessed using the cell counting kit- 8 assay. (C) Following pretreatment with DMSO, Jas or Cyto D, HSC-T6 cells were re-seeded into plastic wells for $1 \mathrm{~h}$ at $37^{\circ} \mathrm{C}$. Following removal of nonadherent cells by washing with phosphate-buffered saline, adherent cells were counted and analyzed. (D) Cell migration was tested in a modified Boyden chamber assay. Following treatment, HSC-T6 cells $\left(1 \times 10^{5}\right)$ were placed in the upper layer. The lower chamber was filled with medium and incubated for $16 \mathrm{~h}$. The migrated cells were stained with DAPI and analyzed. (E) The apoptotic cells were quantified by fluorescence-activated cell sorting following annexin V-FITC and PI staining. Annexin V-positive and PI-negative cells were defined as apoptotic cells. Data are expressed as the mean \pm standard error of three different experiments. "P<0.05. FITC, fluorescein isothiocyanate; Cyto D, cytochalasin D; Jas, jasplakinolide; PI, propidium iodide; EdU, 5'-ethynyl-2'-deoxyuridine; DMSO, dimethylsulfoxide; OD, optical density.

Actin cytoskeleton reorganization-induced HSC-T6 cell activation is associated with the p38 MAPK pathway. Both extracellular signal-regulated kinase (ERK) and p38 MAPK have been shown to regulate HSC activation $(14,15)$. To explore whether those signaling molecules were involved in the actin cytoskeleton reorganization-induced HSC activation,
HSC-T6 cells were pre-incubated for $30 \mathrm{~min}$ with inhibitors of ERK (PD98059) or p38 MAPK (SB203580) prior to treatment with Jas. qPCR revealed that PD98059 did not affect the expression of $\alpha$-SMA or collagen type 1 induced by Jas. By contrast, inhibition of p38 MAPK by SB203580 significantly reduced the Jas-induced expression of $\alpha$-SMA and collagen type 1 (Fig. 4A). 
A

$\alpha-S M A$

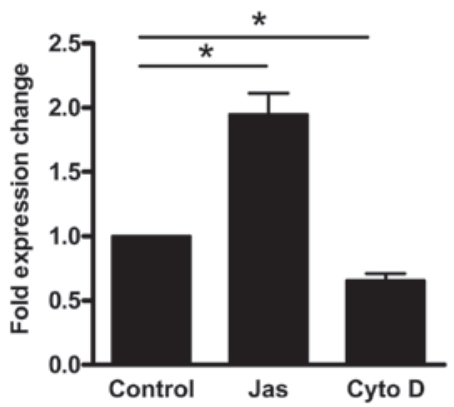

collagen 1

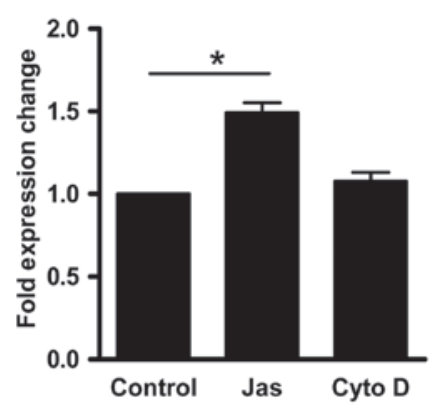

B

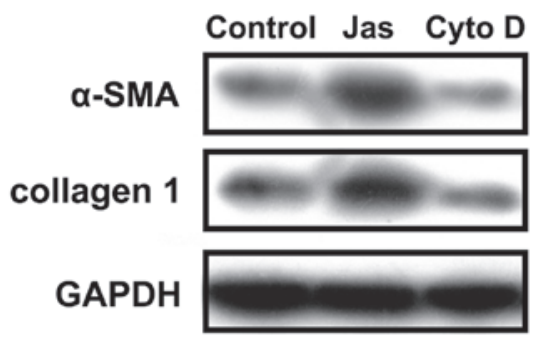

$\alpha-$ SMA

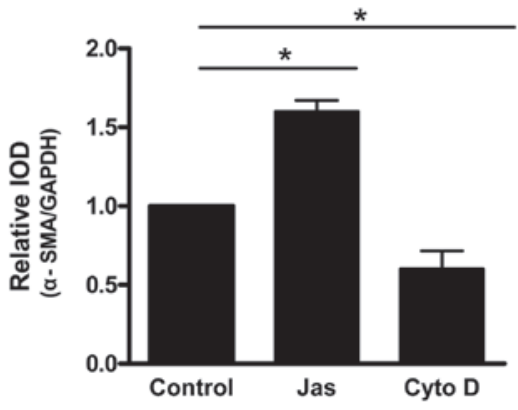

collagen 1

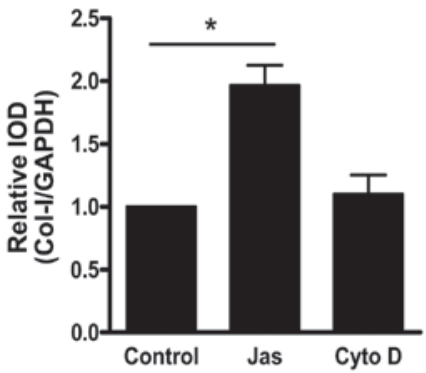

Figure 3. Effect of the actin cytoskeleton reorganization induced by Jas or Cyto D on the activation of HSC-T6 cells. (A) HSC-T6 cells were treated with dimethylsulfoxide, Jas or Cyto D, respectively. The gene expression of $\alpha$-SMA and collagen type 1 was assessed by the quantitative polymerase chain reaction. Data were analyzed using the $2^{-\Delta \Delta C t}$ method. (B) The protein expression of $\alpha$-SMA and collagen type 1 was assessed by western blot analysis. Following treatment, cell lysates were resolved using $12 \%$ SDS-PAGE, followed by transfer to a polyvinylidene fluoride membrane. Western blot analysis was performed with specific antibodies and each band was detected using enhanced chemoluminescence reagent. Data are expressed as the mean \pm standard error of five experiments. "P<0.05. $\alpha$-SMA, $\alpha$-smooth muscle actin; IOD, integrated optical density; Cyto D, cytochalasin D; Jas, jasplakinolide.

A

a-SMA

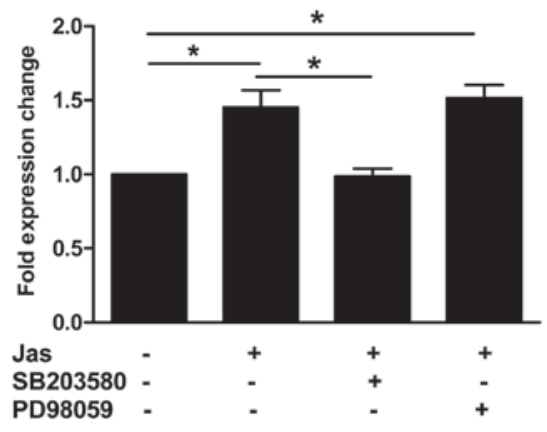

B

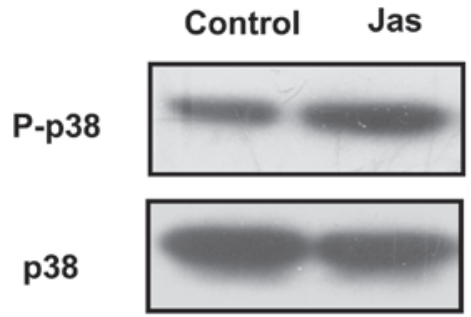

collagen 1
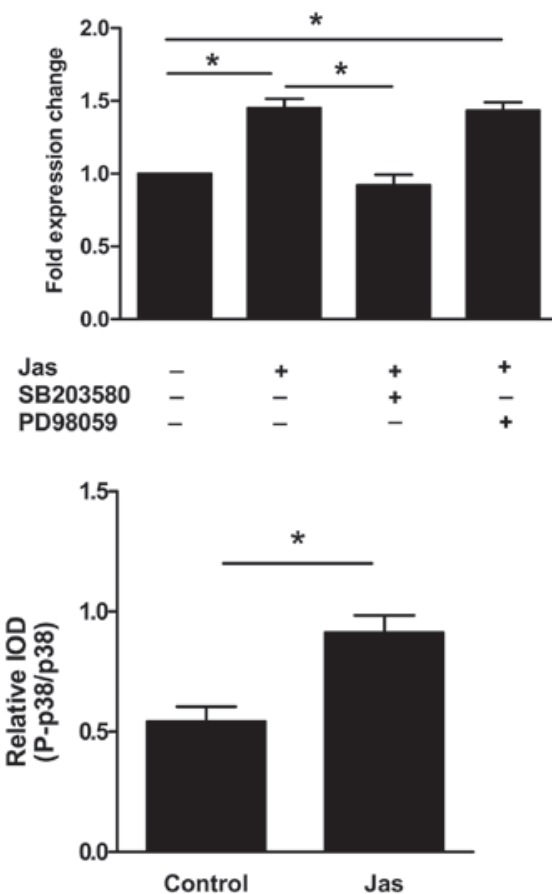

Figure 4. Actin cytoskeleton reorganization-induced HSC-T6 cell activation correlates with the p38 MAPK pathway. (A) HSC-T6 cells were pretreated with PD98059 (10 $\mu \mathrm{mol} / \mathrm{l})$ or SB203580 (100 nmol/l), respectively, for $30 \mathrm{~min}$. The cells were then exposed to DMSO or Jas. Gene expression of $\alpha$-SMA and collagen type 1 was then determined using the quantitative polymerase chain reaction. (B) The phosphorylation status of p-38 MAPK was assessed by western blot analysis. HSC-T6 cells were incubated with DMSO or Jas for $1 \mathrm{~h}$. Cell lysates were resolved using 12\% SDS-PAGE, followed by transfer to a polyvinylidene fluoride membrane. Western blot analysis was performed with specific antibodies to distinguish between different phosphorylation statuses of p38 MAPK. In addition, total p38 MAPK was analyzed as a loading control. P-p38 MAPK was densitometrically analyzed and normalized to total p38 MAPK. The results are expressed as the mean \pm standard error of five experiments. "P<0.05. $\alpha$-SMA, $\alpha$-smooth muscle actin; IOD, integrated optical density; Jas, jasplakinolide; DMSO, dimethylsulfoxide; p38 MAPK, p38 mitogen-activated protein kinase; P-p38, phosphorylated p38 MAPK. 
Since p38 MAPK is critical for the actin cytoskeleton reorganization-induced HSC activation, its activation in HSC-T6 cells treated with Jas was further evaluated. Western blot analysis showed that Jas significantly increased the protein levels of phospho-p38 in HSC-T6 cells (Fig. 4B).

\section{Discussion}

As described previously in this study, during the development of liver fibrogenesis, HSCs undergo a response known as activation, which is the transition of quiescent cells into proliferative, fibrogenic and contractile myofibroblasts (16). However, the mechanism by which HSCs are activated is unclear. Recently, a study by Rombouts et al (5) indicated that the acquisition of certain properties by activated HSCs is highly dependent on the reorganization of the actin cytoskeleton (4). The present study provided direct evidence showing that changes in the actin cytoskeleton, including the assembly of stress fibers, affects the activation of HSCs. As shown in Fig. 1, the F-actin staining of HSCs showed a variation in the intracellular distribution of F-actin between cells incubated with Jas and those incubated with Cyto D. Jas treatment resulted in thick actin bundles and a patchy appearance in the cytoplasm of HSCs. By contrast, Cyto D-treated cells typically exhibited dissolution of actin stress fibers and decreased fluorescent staining. In parallel, the polymerization of actin microfilaments by Jas led to HSC activation: i) Jas upregulated the expression of $\alpha$-SMA and collagen type 1; ii) stabilization of F-actin by Jas improved the migration and adhesion properties of HSCs. Furthermore, the HSC-T6 cell activation induced by the actin cytoskeleton reorganization was associated with the p38 MAPK pathway.

Ikeda et al (17) demonstrated that an aberrant actin cytoskeleton in mice deficient for destrin (an actin depolymerizing factor) was able to cause cell hyperproliferation. Furthermore, the ability of the ECM to modulate cell growth may be mediated partly by the assembly and disassembly of F-actin filaments (18). Although exposure to Jas did not significantly affect the proliferative activity of HSCs in the present study, the cell proliferation was decreased in the Cyto D-treated group. The results also indicate that F-actin has an important role in the regulation of HSC proliferation.

It is well accepted that F-actin is crucial in determining cell shape and migration, as well as in controlling apoptosis (19). Consistent with this, the stabilization of actin by Jas in the present study was observed to significantly augment the migration and adhesion of HSCs, which, by contrast, were impaired by Cyto D. However, F-actin rearrangement appeared to have no effect on the apoptosis of HSCs.

Since the increased expression of $\alpha$-SMA is considered to be a major marker of the activation of HSCs, the association between F-actin and the expression of $\alpha$-SMA was examined. The stabilization of actin by Jas significantly increased the expression of $\alpha$-SMA in HSCs, indicating that F-actin is involved in the activation process of HSCs. Activated HSCs are known to increase the synthesis/secretion of fibrogenic ECM components, including collagen types 1, 3, 5 and 6 (20-22). A study has reported that the expression of collagen type 3 is more significant at the early stages of fibrosis (mild activated HSCs), while collagen type 1 is more significant at the advanced stages (intensive activated HSCs) (23). In the present study, it was found that the activation of HSCs promoted via the stabilization of actin by Jas produced increased levels of collagen type 1. It was thus confirmed that the polymerization of F-actin led to the activation of HSCs. Moreover, the activation of HSCs induced by cytoskeletal reorganization was found to be dependent on p38 MAPK, since SB203580, a p38 MAPK inhibitor, inhibited the gene and protein expression of $\alpha$-SMA and collagen type 1 induced by treatment with Jas.

The findings of the present study may be of importance since they not only indicate that actin reorganization has a pivotal role in regulating the biological functions of HSCs, including cell adhesion and migration, but also provide further insights into the possible molecular mechanisms behind the activation of HSCs. The inhibition of F-actin reorganization may thus be a key factor or molecular target for the control of liver fibrosis or cirrhosis.

\section{Acknowledgements}

The present study was supported by the Natural Science Foundation of Shandong Province (nos. ZR2010HQ046, ZR2011CQ030 and ZR2010DM010), the Program for New Century Excellent Talents in University (no. NCET-10-0922), the National Natural Science Foundation of China (nos. 30900290 and 31270993), the Foundation of Shandong Educational Committee (nos. J09LF06 and J11LF17) and Weifang Science and Technology Development Plan Project (no. 201201282). The authors would like to thank Dr Emil Avsar for the critical reading of the manuscript.

\section{References}

1. Moreira RK: Hepatic stellate cells and liver fibrosis. Arch Pathol Lab Med 131: 1728-1734, 2007.

2. Priya S and Sudhakaran PR: Cell survival, activation and apoptosis of hepatic stellate cells: modulation by extracellular matrix proteins. Hepatol Res 38: 1221-1232, 2008.

3. Jia YL, Shi L, Zhou JN, et al: Epimorphin promotes human hepatocellular carcinoma invasion and metastasis through activation of focal adhesion kinase/extracellular signal-regulated kinase/matrix metalloproteinase-9 axis. Hepatology 54: 1808-1818, 2011.

4. Odena G and Bataller R: Actin-binding proteins as molecular targets to modulate hepatic stellate cell proliferation. Focus on 'MARCKS actin-binding capacity mediates actin filament assembly during mitosis in human hepatic stellate cells'. Am J Physiol Cell Physiol 303: C355-C356, 2012.

5. Rombouts K, Mello T, Liotta F, et al: MARCKS actin-binding capacity mediates actin filament assembly during mitosis in human hepatic stellate cells. Am J Physiol Cell Physiol 303: C357-C367, 2012.

6. Yee HF Jr: Rho directs activation-associated changes in rat hepatic stellate cell morphology via regulation of the actin cytoskeleton. Hepatology 28: 843-850, 1998.

7. Yamazaki D, Kurisu S and Takenawa T: Regulation of cancer cell motility through actin reorganization. Cancer Sci 96: 379-386, 2005.

8. Vogel S, Piantedosi R, Frank J, et al: An immortalized rat liver stellate cell line (HSC-T6): a new cell model for the study of retinoid metabolism in vitro. J Lipid Res 41: 882-893, 2000.

9. Herrmann J, Gressner AM and Weiskirchen R: Immortal hepatic stellate cell lines: useful tools to study hepatic stellate cell biology and function? J Cell Mol Med 11: 704-722, 2007.

10. Zhang X, Cui X, Cheng L, et al: Actin stabilization by jasplakinolide affects the function of bone marrow-derived late endothelial progenitor cells. PLoS One 7: e50899, 2012.

11. Heidkamp MC, Bayer AL, Scully BT, Eble DM and Samarel AM: Activation of focal adhesion kinase by protein kinase $\mathrm{C}$ epsilon in neonatal rat ventricular myocytes. Am J Physiol Heart Circ Physiol 285: H1684-H1696, 2003. 
12. Salguero Palacios R, Roderfeld M, Hemmann S, et al: Activation of hepatic stellate cells is associated with cytokine expression in thioacetamide-induced hepatic fibrosis in mice. Lab Invest 88: 1192-1203, 2008.

13. Issa R, Zhou X, Trim N, et al: Mutation in collagen-1 that confers resistance to the action of collagenase results in failure of recovery from CCl4-induced liver fibrosis, persistence of activated hepatic stellate cells, and diminished hepatocyte regeneration. FASEB J 17: 47-49, 2003.

14. Chen A and Zheng S: Curcumin inhibits connective tissue growth factor gene expression in activated hepatic stellate cells in vitro by blocking NF-kappaB and ERK signalling. Br J Pharmacol 153: 557-567, 2008.

15. Zhou Y, Jia X, Wang G, Wang X and Liu J: PI-3 K/AKT and ERK signaling pathways mediate leptin-induced inhibition of PPARgamma gene expression in primary rat hepatic stellate cells. Mol Cell Biochem 325: 131-139, 2009.

16. Friedman SL: Molecular regulation of hepatic fibrosis, an integrated cellular response to tissue injury. J Biol Chem 275: 2247-2250, 2000.
17. Ikeda S, Cunningham LA, Boggess D, et al: Aberrant actin cytoskeleton leads to accelerated proliferation of corneal epithelial cells in mice deficient for destrin (actin depolymerizing factor). Hum Mol Genet 12: 1029-1037, 2003.

18. Nagano N, Aoyagi M, Hirakawa K, Yamamoto $M$ and Yamamoto K: Organization of F-actin filaments in human glioma cell lines cultured on extracellular matrix proteins. J Neurooncol 27: 215-224, 1996 .

19. Barbier S, Chatre L, Bras M, et al: Caspase-independent type III programmed cell death in chronic lymphocytic leukemia: the key role of the F-actin cytoskeleton. Haematologica 94: 507-517, 2009.

20. Hernandez-Gea V and Friedman SL: Pathogenesis of liver fibrosis. Annu Rev Pathol 6: 425-456, 2011.

21. Lee UE and Friedman SL: Mechanisms of hepatic fibrogenesis. Best Pract Res Clin Gastroenterol 25: 195-206, 2011.

22. Senoo H, Yoshikawa K, Morii M, Miura M, Imai K and Mezaki Y: Hepatic stellate cell (vitamin A-storing cell) and its relative - past, present and future. Cell Biol Int 34: 1247-1272, 2010.

23. Wang LT, Zhang B and Chen JJ: Effect of anti-fibrosis compound on collagen expression of hepatic cells in experimental liver fibrosis of rats. World J Gastroenterol 6: 877-880, 2000. 